\title{
ACCURACY OF DIAGNOSIS OF INTESTINAL HELMINTH PARASITES IN A REFERENCE DIAGNOSTIC LABORATORY IN THE ASHANTI REGION OF GHANA
}

\author{
SAMMY C.K. TAY ${ }^{1}$, STEPHEN Y. GBEDEMA ${ }^{2 *}$, THOMAS K. GYAMPOMAH ${ }^{3}$ \\ ${ }^{1}$ Department of Clinical Microbiology, School of Medical Sciences, College of Health Sciences, Kwame Nkrumah \\ University of Science and Technology, Kumasi, Ghana. \\ 2Department of Pharmaceutics, Faculty of Pharmacy and Pharmaceutical Sciences, College of Health Sciences, \\ Kwame Nkrumah University of Science and Technology, Kumasi, Ghana. \\ ${ }^{3}$ Department of Clinical Microbiology, Komfo Anokye Teaching Hospital, Kumasi, Ghana \\ ${ }^{*}$ Corresponding author. E-mail: sgbedema.pharm@knust.edu.gh
}

Received: January 31, 2011; Accepted: February 15, 2011

\begin{abstract}
There are reports of many intestinal-helminth negative stool specimens in most laboratories in Ghana even though the prevalence of these infections seems to be on the increase. In this study 2000 stool specimens collected between May and October 2008 in The Reference Laboratory of the Ashanti Region of Ghana were screened using Direct Wet Mount, Kato-Katz and Formol-Ether Concentration methods. The Formol-Ether Concentration method gave the highest prevalence (11.1\%) of helminth parasites made up of hookworm, Dicrocoelium dendriticum, Strongyloides stercoralis, Schistosoma mansoni, Hymenolepis nana, Taenia species and Trichuris trichiura. The direct wet mount and Kato-Katz detected total prevalence of $3.2 \%$ and $5.1 \%$ respectively. Kato-Katz showed good agreement with the formol-ether concentration in the detection of hookworms, T. trichiura and S. mansoni infections ( $99.1 \%$ sensitivity) with positive and negative predictive values of $100 \%$ and $99.5 \%$ respectively. This study confirmed earlier reports that the traditional Direct Wet Mount screening test is less sensitivity hence the employment of Formol-Ether Concentration as a confirmatory test in routine laboratory examination of stool samples will significantly reduce misdiagnosis of intestinal helminth infections and its attendant public health consequences.
\end{abstract}

Key Words: diagnosis, formol-ether concentration, Kato-Katz, direct wet mount.

\section{INTRODUCTION}

Intestinal helminth infection is one of the most prevalent and widespread chronic infections of man [1] with over a quarter of the world's population being infected. Roundworm, hookworm and whipworm infections are estimated at 1.47, 1.05, and 1.30 billion people respectively [2,3]. Many intestinal helminths trigger immune responses in man and these may drain the body's ability to fight other diseases, making affected individuals more prone to co-infection [4]. There are reports that helminthiasis is contributing to the unrelenting prevalence of AIDS and tuberculosis in many developing countries particularly in Africa [5]. The majority of soil-transmitted helminth infections are found in the third world countries as a result of low standard of living due to poor personal hygiene and environmental sanitation $[6,7,8,9]$. In many West African communities, prevalence of helminth infections exceeds 70\% [10]. School-age children are particularly vulnerable to these parasites because of their high mobility and lower standards of hygiene [11]. In Ghana, Annan et al. [12] reported up to $63 \%$ of intestinal helminth infections in school-age children. Nevertheless, the levels of helminth infections diagnosed in hospital laboratories in Ghana are very low and do not highlight the high prevalence as reported by many researchers in the sub-region [13]. This study seeks to find out if there is some critical diagnostic factor responsible for the low recovery of helminth parasites from stool specimens.

\section{MATERIALS AND METHODS}

Ethical issues: The study protocol was approved by the Committee on Human Research, Publications and Ethics, School of Medical Sciences, Kwame Nkrumah University of Science and Technology in collaboration with the Reference Laboratory of the Ashanti Region of Ghana management which also granted the permission to undertake the study.

Study area and specimen collection: The study was carried out in Kumasi, Ghana. A non-selective sample of 2000 consented in- and out-patients aged 
between 1 - 90 years were recruited for the study between May 2008 to October 2008. Specimen collection and handling were carried out in accordance with standard protocols reported by Booth et al. [14], NCCLS [15], Melvin and Brooke [16] and WHO [17].

Parasitologic examination: The direct wet mount, Kato-Katz and Formol-ether concentration, methods were employed. Each specimen was first examined macroscopically and its consistency or nature was recorded in accordance with the description by Ash and Orihel [18], Estevez and Levine [19] and Smith and Schad [20]. The test procedures were carried out in accordance with standard protocols as described by Garcia $[21,22]$ and WHO $[17,23]$.

Helminth Infection intensity determination by Kato Katz technique: The faecal sample was pressed through a Kato nylon screen mesh (HelmR test kits, Belo Horizonte, Brazil) of size $200 \mu \mathrm{m}$ and the sieved stool was transferred into the $6 \mathrm{~mm}$ diameter hole of the cardboard template on a microscope slide. The template was then removed and the remaining sample (approximately $42 \mathrm{mg}$ ) was covered with the glycerol soaked cellophane strip of size $25 \times 35 \mathrm{~mm}$ and 50 um thick. The prepared slide was microscopically examined and the eggs of each of the helminth species were recorded. The number of eggs per gram of faeces was calculated and used to estimate the infection intensity of the parasites based on the classification reported by WHO (2002) for major soil-transmitted helminths and S. mansoni infections. Loose and watery stool specimens were not analyzed by the Kato-Katz method because of the technical difficulties that limit analysis of such specimens by this method $[10,25]$.

Helminth infection intensities The intensity of helminth infection was measured on the basis of egg counts per gram (epg) of stool [26], determined by the Kato-Katz method. Infection intensities for the soil transmitted helminths (STHs) and $S$. mansoni cases detected in the study were classified in accordance with the criteria prescribed by $\mathrm{WHO}$ [24]. All cases of STHs found in the study were of low intensity. It was evidenced that a significantly high number of individuals infected with medium and high S. mansoni burden belonged to $10-15$ age group $(p<0.05$; Table 4$)$.

\section{RESULTS AND DISCUSSION}

Routine diagnosis of intestinal helminth infections is normally based on detection of the helminth eggs and/or larvae in stool samples examined through a variety of parasitologic methods [27]. In most laboratories in Ghana and many other developing nations, the Direct Wet Mount is the preferred stool parasitological detection technique because it is simple and fast to perform and the most affordable $[21,22,28,29,30,31]$.

The study showed that 506 (25.3\%) out of the 2000 patients had provisional diagnoses that could be related to intestinal parasitic diseases (Table 1) and most of the helminth-positive specimens (75\%) were from these patients. On the other hand the laboratory tests requested for $30.4 \%$ of the population studied had no provisional diagnosis or clinical summaries related to the investigations ordered. Most of the stool specimens (95\%) from these patients tested negative for helminth parasites, which implied that the request for these stool examinations were probably inappropriate and did not significantly $(p<0.05)$ contribute to patient care [32].

The total prevalence of intestinal helminth infections detected by the formol-ether concentration, KatoKatz and the direct wet mount methods were $11.1 \%, 5.2 \%$ and $3.25 \%$, respectively. The direct wet mount exhibited the lowest performance (29.2\%); being about three times less sensitive than the formol-ether concentration (Table 2). Kato-Katz method $(43.4 \%)$ is generally about twice as less sensitive than the formol-ether concentration (Fig. 1) but exhibited comparable sensitivity in the detection of hookworm, T.trichiura and S. mansoni (Table 3).

The differences $(p<0.05)$ observed in the sensitivities of the three screening methods have been reported in other studies [27,33,34,35,36,37]. This study therefore appears to unveil underestimation of helminth infection prevalence in this diagnostic laboratory and other medical laboratories in Ghana since the direct wet mount method is the commonest employed helminth parasitological detection test in the country. The study also highlighted a high occurrence of low worm burden (Table 4) among the population which cannot be easily detected by the direct wet mount technique.

The Kato-Katz exhibited very low sensitivity for the detection of intestinal helminth parasites such as $S$. stercoralis, Dicrocoelium species, Taenia species and $H$. nana. Similar observations have been reported elsewhere $[33,35,38,39,40]$. The choice of the direct wet mount over the Kato-Katz method is desirable when there is a good indication of infection with S. stercoralis, Dicrocoelium species, Taenia species and $H$. nana. At any rate, the use of the formol-ether concentration as a confirmatory test will significantly reduce misdiagnosis of intestinal helminth infections and its attendant public health consequences.

\section{CONCLUSION}

The study has shown that the intensity of intestinal helminth infections reported at the Reference 
Laboratory were mostly of low grade and can only efficiently be detected by the formol-ether concentration method which exhibited superior sensitivity to Kato Katz technique and the traditional Direct Wet Mount method. The employment of formol-ether concentration technique as a confirmatory test in routine laboratory examination of stool will significantly reduce misdiagnosis of intestinal helminth infections and its attendant public health consequences.

\section{ACKNOWLEDGEMENT}

The authors will like to appreciate the technical support offered by the staff of the Parasitology and Clinical Microbiology Laboratories of the School of Medical Sciences, KNUST, and the Nuguchi Memorial Institute, University of Ghana.

\section{REFERENCES}

[1] Stoll N.R. (1999) J. Parasitol. (85):392396.

[2] Bethony J., Brooker S., Albonico M., Geiger S.M., Loukas A., Diemert D. and Hotez P.J. (2006) Lancet 367: 15211532.

[3] Van der Werf M.J., de Vlas S.J., Brooker S., Looman C.W., Nagelkerke N.J., Habbema J.D. and Engels D. (2003) Acta Tropica (86): 125 - 39.

[4] Watkins W.E. and Pollitt E. (1997) Psychological Bulletin 121 (2) 171-191.

[5] Borkow G. and Bentwich Z. (2000) Bulletin of World Health Organization, 78(11).

[6] Oduntan S.O. (1974) Annals of Tropical Medicine and Parasitology, (68):145156.

[7] Bundy D.A.P. and Guyatt H.L. (1996) Parasitology Today 12: 1-16.

[8] Hasegawa H., Miyagi I., Kamimura K., Nainggolan I.J.J., Tumewu-Wagei M. (1992) Southeast Asian J Trop Med Pulbic Health (2): 87-9.

[9] Feachem R.G., Guy M.W., Harrison S., Iwugo K.O., Marshall T., Mbere N., Muller R. and Wright A.M. (1983) Transactions of the Royal Society of Tropical Medicine and Hygiene 77, 515-521.

[10] Brooker S., Marriot H., Hall A., Adjei S., Allan E., Maier C., Bundy D. A., Drake L. J., Coombes M. D., Azene G., Lansdown R. G., Wen S. T., Dzodozmenyo M., Cobbinah J., Obro N., Kihamia C. M., Issae W., Mwanri L., Mweta M. R., Mwaikemwa A., Salimu M., Ntimbwa P., Kiwelu V. M., Turuka A., Nkungu D. R.,Magingo J. (2001) Tropical Medicine and International Health 6, 1075-1083.
[11] Fentiman A., Hall A.,Bundy D. (2001) Social Science and Medicine 52, 429439.

[12] Annan A., Crompton D.W.T., Walters D.E., Arnold S.E. (1986) Parasitology 92: 209-217.

[13] Wirkom V., Tata R., Agba M., Nwobu G., Ndze R., Onoja O., Utien G., Bongkisheri L., Nsadzetreno V. and Banseka E. (2007) The Internet Journal of Tropical Medicine. Vol. 5 No.1:1-8.

[14] Booth M., Vounatsou P., N'Goran E.K., Tanner M. and Utzinger J. (2003) Parasitology 127 pp. 525-531.

[15] NCCLS (1997). Procedures for the Recovery and Identification of Parasites from the Intestinal Tract. Approved guideline M28-A. National Committee for Clinical Laboratory Standards, Wayne, $\mathrm{Pa}$.

[16] Melvin D.M. and Brooke M.M. (1985) . Laboratory Procedures for the Diagnosis of Intestinal Parasites, $p$. 163-189. U.S. Department of Health, Education, and Welfare publication no. (CDC) 85-8282. U.S. Government Printing Office, Washington, D.C.

[17] World Health Organization (1991) Basic Laboratory Methods in Medical Parasitology. Geneva: World Health Organization. Geneva.

[18] Ash L.R., Orihel T.C. (1991) Parasites: A Guide to Laboratory Procedures and Identification. Chicago: American Society of Clinical Pathologists.

[19] Estevez E.G. and Levine J.A. (1985) J Clin Microbiol (22): 666-667.

[20] Smith G., Schad G.A. (1990) Parasitology.(99): 127-132.

[21] Garcia L.S. (1999) Practical Guide to Diagnostic Parasitology. ASM Press, Washington, D.C.

[22] Garcia L.S. (2001) Diagnostic Medical Parasitology, 4th ed. ASM Press, Washington, D.C.

[23] World Health Organization, WHO (1994) Bench aids for the diagnosis of intestinal parasites. World Health Organisation, Geneva.

[24] World Health Organization (2002). www.who.int/ctd/para/disease.php.

Accessed November 20, 2008. Prevention and Control of Schistosomiasis and Soil-Transmitted Helminthiasis. WHO Technical Series Report 912. Geneva.

[25] Siegel D.L., Edelstein P.H. and Nachamkin I. (1990) JAMA 263:979982. 
[26] Beaver P.C. (1950) J. Parasitol;; 36: 451-6

[27] Goodman D., Haji H.J., Bickle Q.D., Stoltzfus R.J., Tielsch J.M., Ramsan M., Savioli L. and Albonico M. (2007) Am. J. Trop. Med. Hyg. (76): 725-731.

[28] Cheesbrough M. (2005) ed., Parasitological test in: District Laboratory Practice in Tropical Countries, Part 1, Tropical Health Technologies, Cambridge, pp178 -306.

[29] Arcari M., Boxendine A. and Bennett C.E. (2000) Diagnosis medical parasites through coprological techniques. Available online at: http//www.soton.ac.uk/ceb/diagnosis/v. Accessed November 20, 2008.

[30] Bogoch I.I., Raso G., N'Goran E.K., Marti H.P., Utzinger J. (2006) Eur J Clin Microbiol Infect Dis 25: 344-347.

[31] De Kaminsky R.G. (1993) J Parasitol 79: 277-280.

[32] Grimshaw J., Russel I. (1993) Lancet (342): 1317-1322.
[33] Martin L.K. and Beaver P.C. (1968) Amer. J. Trop. Med. \& Hyg. (17): 382389.

[34] Watson B., Blitzer M., Rubin $H_{\text {., }}$ Nachamkin I. (1988) Am J Clin Pathol (89): 389-391.

[35] Engels D., Nahimana S., Gryseels B. (1996) Transactions of the Royal Society of Tropical Medicine and Hygiene (90): 523-25.

[36] Akujobi C.N., Iregbu K.C. and Odugbemi T.O. (2005) Nigerian Journal of Health and Biomedical Sciences. 4(1): 5-7.

[37] Oguama V.M. and Ekwunife C.A. (2007) The Internet journal of Tropical Medicine. Vol. 3 no. 2.

[38] Kato K. \& Miura M. (1954) Japanese Journal of Parasitology (3): 35-37.

[39] Katz N., Chaves A. \& Pellegrino J. (1972) Revista do Instituto de Medicina Tropical de Sao Paulo 14, 397-400.

[40] Ebrahim A., El-Morshedy H., Omer E., El-Daly S., Barakat R. (1997) Am J Trop Med Hyg 57: 706-708.

Table 1- Provisional clinical diagnosis of patients studied for helminth infections at KATH

\begin{tabular}{lc}
\hline Clinical diagnosis & Frequency \\
\hline Anaemia & 187 \\
Abdominal pain & 83 \\
Diarrhoea & 77 \\
Helminthiasis & 56 \\
Gastroenteritis & 45 \\
Dysentery & 38 \\
Nausea & 20 \\
Antenatal care & 775 \\
Medical Examinations & 112 \\
${ }^{*}$ No diagnosis indicated & 536 \\
Others & 71 \\
\hline Total & 2000 \\
\hline$\quad$ Key: & \\
$\quad *=$ patients with no provisional diagnosis related to the investigations requested. \\
$\quad$ † included malaria, enteric fever, urinary tract infection and chronic kidney and liver diseases.
\end{tabular}

Table 2- Performance of Direct wet mount test against formol-ether concentration in detection of intestinal helminth parasites

\begin{tabular}{clccc}
\hline Method & & \multicolumn{2}{c}{ Direct wet mount test } & Total Results \\
\hline Formol-ether & Results & Positive & Negative & \\
\cline { 2 - 4 } concentration & Positive & 66 & 160 & 226 \\
test & Negative & 0 & 1774 & 1774 \\
\hline Total results & & 66 & 1934 & $\mathbf{2 0 0 0}$ \\
\hline
\end{tabular}


Table 3- Comparison of Kato- Katz versus Formol-ether concentration tests for detection of intestinal helminth parasites.

\begin{tabular}{lcc}
\hline Parasite & Formol-ether concentration & Kato-Katz \\
\hline Hookworm & 58 & 54 \\
T. trichiura & 2 & 2 \\
A. lumbricoides & 0 & 0 \\
S. mansoni & 37 & 34 \\
\hline Total results & $\mathbf{9 7}$ & $\mathbf{9 0}$
\end{tabular}

Table 4- Infection Intensity of STHs and S. mansoni using the Kato-Katz

\begin{tabular}{lcccc}
\hline Helminth & \multicolumn{3}{c}{ Infection Intensity (\%) } & \multirow{2}{*}{$\begin{array}{c}\text { Total positive by } \\
\text { Kato Katz (\%) }\end{array}$} \\
\cline { 2 - 4 } & Low & Moderate & Heavy & \\
\hline Hookworm & $54(60.0)$ & $0(0.0)$ & $0(0.0)$ & $54(60.0)$ \\
Trichuris trichura & $2(2.2)$ & $0(0.0)$ & $0(0.0)$ & $2(2.2)$ \\
Schistosoma mansoni & $14(15.6)$ & $16(17.8)$ & $4(4.4)$ & $34(37.8)$ \\
\hline Total Results & $70(77.8)$ & $16(17.8)$ & $4(4.4)$ & $90(100)$ \\
\hline
\end{tabular}

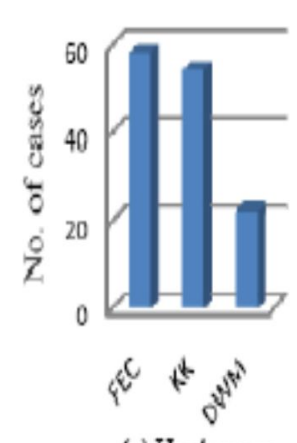

(a) Hookworm

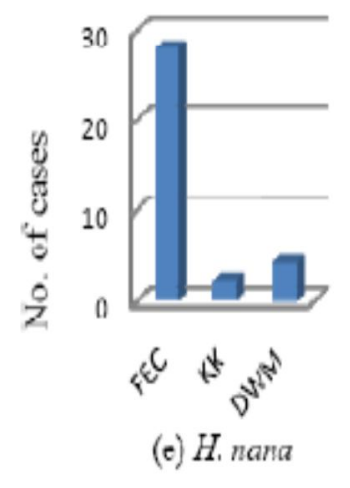

(e) H. nana
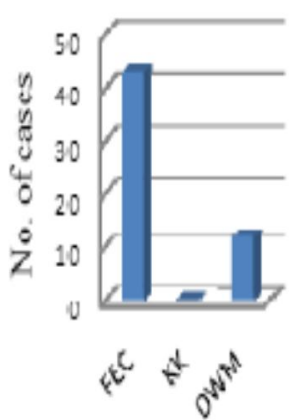

(b) D. dendrutcum

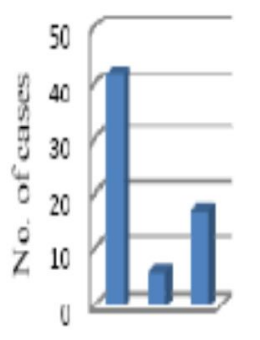

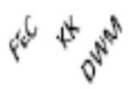

(c) S. stercoralis:

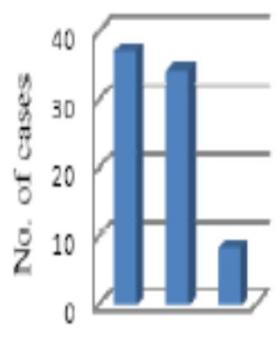

\& 40

(d) S. mansoni
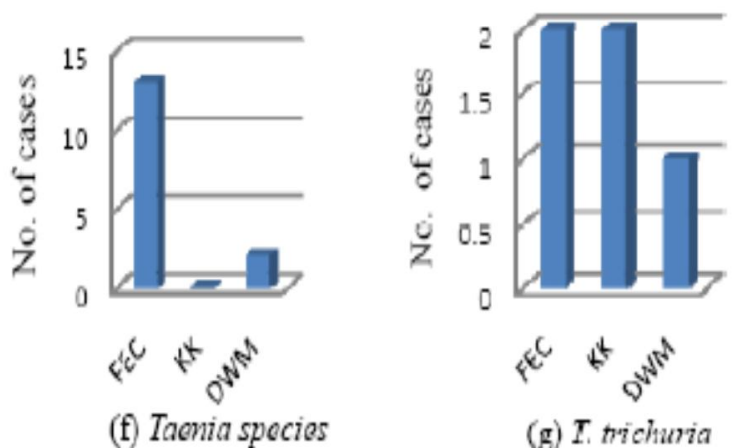

Fig. 1. Prevalence of intestinal helminths as detected by the various techniques (FEC- Formolether concentration; KK- Kato Katz; DWM- Direct wet mount). 